\title{
Deleterious BRCA1/2 mutation is an independent risk factor for carboplatin hypersensitivity reactions
}

\author{
D H Moon ${ }^{1,2,3}, \mathrm{~J}-M$ Lee ${ }^{* 1,3}$, A M Noonan ${ }^{1}$, C M Annunziata ${ }^{1}$, L Minasian ${ }^{1}$, N Houston ${ }^{1}$, J L Hays $^{1}$ \\ and E C Kohn ${ }^{1}$ \\ ${ }^{1}$ Medical Oncology Branch, Center for Cancer Research, National Cancer Institute, National Institutes of Health, Bethesda, \\ MD, USA and ${ }^{2}$ Medical Research Scholars Program, National Institutes of Health, Bethesda, MD, USA
}

Background: We tested the hypothesis that BRCA1/2 mutation carriers with ovarian cancer are at higher risk of carboplatin hypersensitivity reactions (HSRs).

Methods: Medical records of women enrolled in two carboplatin + olaparib clinical trials (NCT01237067/NCT01445418) were reviewed. A maximum of eight cycles containing carboplatin were administered.

Results: All women $(\mathrm{N}=87)$ had good performance status and end-organ function. Incidences of carboplatin HSR before enrolment and on study were $17 \%$ and $21 \%$, respectively. Most patients who developed carboplatin HSR had a deleterious BRCA1/2 mutation (93\%) vs 50\% in patients without HSR $(P<0.0001)$. Multivariable analysis accounting for potential confounding variables including age, history of allergies, and cumulative prior carboplatin cycles confirmed deleterious BRCA1/2 mutation as an independent risk factor for carboplatin HSR (odds ratio 13.1 (95\% confidence interval 2.6-65.4), $P=0.0017$ ). Mutation carriers had onset of carboplatin HSR at lower cumulative exposure $(P=0.003)$. No significant difference in outcome was observed on our study between patients with and without a history of HSR.

Conclusion: Deleterious BRCA1/2 mutation increased susceptibility and shortened time to carboplatin HSR, independently of other reported factors. These data suggest that at-risk women should be counselled regarding likelihood, symptoms, and potential earlier onset of carboplatin HSRs.

Cisplatin and carboplatin, elemental platinum-based chemotherapeutic agents, are cornerstones of gynaecologic cancer treatment. Comparable efficacy and reduced renal and neurotoxicity have led to broad international use of carboplatin. Carboplatin, in combination with paclitaxel, is now the standard front-line treatment for ovarian, tubal, and peritoneal carcinomas, following cytoreductive surgery or in neoadjuvant therapy (Ozols et al, 2003; Vergote et al, 2010); it also is being evaluated in intraperitoneal adjuvant treatment regimens. Carboplatin is the preferred agent in combination regimens for women with platinum-sensitive recurrent ovarian, tubal, and peritoneal cancers. In addition, it is being examined in on-going clinical trial in combination with investigational agents targeting the tumour microenvironment, DNA damage and repair, and/or cell cycle-specific categories, such as angiogenesis inhibitors (Burger et al, 2011) and poly ADP ribose polymerase (PARP) inhibitors.

Carboplatin is generally well tolerated despite its cumulative bone marrow toxicity. Reports of hypersensitivity reactions (HSRs) to carboplatin have increased with the rise in its repetitive use in many cancer patients. Incidence of carboplatin HSR ranges from 8 to 16\% (Markman et al, 1999; Polyzos et al, 2001; Koshiba et al, 2009) and can be as high as $44 \%$ in the second- and third-line

*Correspondence: Dr J-M Lee; E-mail: leej6@mail.nih.gov

${ }^{3}$ These authors contributed equally to the design, execution, analysis, and writing of this project.

Received 13 March 2013; revised 20 June 2013; accepted 25 June 2013; published online 18 July 2013

(C) 2013 Cancer Research UK. All rights reserved 0007-0920/13 
settings (Morgan et al, 1994). Symptoms and signs of this allergic reaction include mild rash to diffuse erythroderma, pruritus, urticaria, angioedema, tachycardia, chest tightness, bronchospasm, dyspnea, and at the severe end, cardiopulmonary collapse. Women who experience moderate-to-severe HSR, defined by lack of or limited improvement of symptoms with interruption of carboplatin infusion (Markman et al, 1999), often have platinum therapy discontinued. Many may not be re-challenged, despite potential clinical benefit and published regimens of premedication and gradual increased drug exposure. Thus, the development of reliable parameters with which to identify those at highest risk for HSR has become an important clinical need.

Deleterious germline BRCA $1 / 2$ mutations (BRCA $1 / 2^{\text {mut }}$ ) account for up to $17 \%$ of the tubo-ovarian cancer population (TCGARNetwork, 2011). These patients have increased therapeutic susceptibility to platinums (Alsop et al, 2012) and thus often receive multiple lines of platinum-based chemotherapy. We hypothesised that this repetitive and potentially higher lifetime platinum exposure may put BRCA mutation carriers with tuboovarian cancer at higher risk for platinum HSR. We examined this hypothesis in our cohort of sporadic high-grade serous and BRCA1/2 ${ }^{\text {mut }}$-positive ovarian cancer patients who received the combination of carboplatin and olaparib, a PARP inhibitor.

\section{MATERIALS AND METHODS}

Patient eligibility and treatment. Eligible patients for this analysis had ovarian, fallopian tube, and primary peritoneal carcinoma, were enrolled in one of two IRB-approved carboplatin + olaparib studies at the Medical Oncology Branch of the National Cancer Institute (NCI; NCT01237067 and NCT01445418), and received at least one attempted infusion of carboplatin. Trial eligibility criteria included recurrent disease, an Eastern Cooperative Oncology Group performance status of $0-2$, adequate endorgan function, and measurable or evaluable disease. Patients with both platinum-refractory and -resistant disease were eligible. All patients had prior platinum treatment and were at least 6 months from their last platinum therapy. Patients with a documented history of life-threatening (grade 4) HSR to carboplatin or cisplatin were excluded from the clinical trials for safety. NCI Common Toxicity Criteria for Adverse Events (CTCAE) 4.0 (Bethesda, MD, USA) was used to characterise and grade adverse events.

Patients received the combination of intravenous carboplatin (AUC 3-5) and oral olaparib (100-400 mg every $12 \mathrm{hr}$ in either capsule or tablet formulation) for a maximum of 8 cycles, followed by olaparib (100-4000-400f intravenous carboplatin (AUC 3-5) aeight cycles, followed by olaparib single-agent maintenance therapy until disease progression. Women with a documented history of mild-to-moderate HSR received premedication with methylprednisolone, ranitidine, and diphenhydramine, and a slowinfusion protocol (Lee et al, 2004). Patients developing carboplatin HSR (grades 1-3) on trial received the premedicated progressive infusion protocol on subsequent carboplatin infusions. If the HSR was grade 4 or recurrent despite premedication and slow progressive infusion, carboplatin was discontinued in favour of olaparib monotherapy.

Data collection. Clinical data collected included demographics, tumour type, stage, prior platinum therapies, medication and environmental factors allergy history, adverse events, and therapy response. Platinum-free interval was defined as the time between the last cycle of the previous platinum chemotherapy and the first carboplatin infusion on trial. Medical records were reviewed to detail carboplatin HSR documented before enrolment, and to characterise carboplatin HSR occurring on trial.
BRCA $1 / 2^{\text {mut }}$ status was obtained as part of the clinical trial enrolment, and sources included the patient's genetic test results, and in one case, deleterious mutation risk outcome estimates using the BRCAPro model (BayesMendel Lab, http://bcb.dfci.harvard. edu/bayesmendel/brcapro.php). BRCAPro $>30 \%$ was defined as a high-risk equivalent by the trial (NCT01445418). Family history was reviewed for eight patients with neither a genetic nor BRCAPro test result to verify the absence of significant family history of breast and ovarian cancer, risk as defined by US Preventive Services Task Force (USPSTF, 2005). These patients were classified as BRCA negative.

Statistical analysis. Summary univariable statistics were reported as medians and ranges for continuous variables, and values and percentages for categorical variables. Cohorts of patients were compared using the Student's $t$-test for continuous variables and Fisher's exact test for categorical variables, to assess for potential predictive factors for carboplatin HSR. Comparisons were twosided assuming unequal variance, and $P$-values of $<0.05$ were considered statistically significant, without adjustment for multiple comparisons. Multivariable logistic regression analyses were conducted to adjust for potential confounders. Kaplan-Meier statistics were used to examine the role of BRCA1/2 ${ }^{\text {mut }}$-associated cumulative risk for carboplatin HSR and the role of HSR status on progression-free survival. All statistical analyses were conducted using SAS (version 9.3; SAS Institute Inc., Cary, NC, USA), with the exception of Kaplan-Meier and log-rank analyses, which were generated using GraphPad Prism (version 5.0d; GraphPad Software, Inc., La Jolla, CA, USA).

\section{RESULTS}

Patient characteristics. Records were analysed for 87 patients with primary diagnoses of high-grade recurrent ovarian, fallopian tube, and primary peritoneal carcinoma who initiated at least one cycle of carboplatin in combination with olaparib per clinical trial protocol (Table 1). The median age was 56 years (range: $27-80$ ) and all patients had received platinum-based therapy before enrolment, almost all in combination with paclitaxel or docetaxel and a few patients with gemcitabine, doxorubicin, or bevacizumab. Patients had received a median of 9 total prior carboplatin cycles, and 12 prior platinum cycles (cisplatin and carboplatin). One patient had received oxaliplatin in combination with cyclophosphamide and gemcitabine before enrolment in the current trial; the number of oxaliplatin cycles could not be ascertained from the medical records and as such, her oxaliplatin cycles were excluded from the platinum cycle calculation. The median platinum-free interval from last platinum exposure was 15.9 months (5.7-82.4). In this cohort, 55 patients had a deleterious BRCA $1 / 2^{\text {mut }}$, and 1 patient had a positive BRCAPro (risk $=68 \%$ ). Thus, 56 out of 87 patients (64\%) had a high-risk BRCA status.

Prior history of carboplatin HSR and HSR on trial. Carboplatin HSR frequency, both before enrolment and on study, was determined. Reaction to carboplatin before trial enrolment was identified in 17\% (15 out of 87) of patients, and ranged from a mild rash or localised pruritus to hypotension and anaphylaxis without life-threatening consequences (grades 1-3). For safety, these patients received premedication and underwent a slow progressive carboplatin infusion as described in Materials and Methods section. Once enrolled, 21\% (18 out of 87 ) of patients developed carboplatin HSR, 22\% (4 out of 18) of whom had a prior history of carboplatin HSR and reacted despite the safety precautions. Thus, the overall incidence of carboplatin HSR at any point in the patient's oncologic history was 33\% (29 out of 87 ). Given that only 4 out of 15 patients with a prior history developed HSR on trial, prior history of carboplatin HSR was not predictive of HSR on 


\begin{tabular}{|c|c|}
\hline & All patients \\
\hline & $\mathbf{N}=87(\% /$ range $)$ \\
\hline Median age & $56(27-80)$ \\
\hline \multicolumn{2}{|l|}{ Tumour type } \\
\hline $\begin{array}{l}\text { Ovarian epithelial } \\
\text { Fallopian tube } \\
\text { Primary peritoneal }\end{array}$ & $\begin{array}{c}80(92.0) \\
2(2.3) \\
5(5.7)\end{array}$ \\
\hline \multicolumn{2}{|l|}{ Stage } \\
\hline $\begin{array}{l}\text { II } \\
\text { III } \\
\text { IV }\end{array}$ & $\begin{array}{c}5(5.7) \\
48(55.2) \\
34(39.1)\end{array}$ \\
\hline \multicolumn{2}{|l|}{ BRCA mutation status ${ }^{a}$} \\
\hline $\begin{array}{l}\text { BRCA1 } \\
\text { BRCA2 } \\
\text { Any BRCA mutation } \\
\text { Negative }\end{array}$ & $\begin{array}{l}44(50.6) \\
12(13.8) \\
56(64.4) \\
31(35.6)\end{array}$ \\
\hline $\begin{array}{l}\text { History of allergy to medications, environmental } \\
\text { factors, foods, and type IV contrast }\end{array}$ & $44(50.6)$ \\
\hline Median platinum-free interval in months & $15.9(5.7-82.4)$ \\
\hline Median number of prior carboplatin regimens & $2(0-6)$ \\
\hline Median number of prior carboplatin cycles & $9(0-42)$ \\
\hline Median number of prior platinum regimens ${ }^{b}$ & $2(1-6)$ \\
\hline Median number of prior platinum cycles ${ }^{b}$ & $12(2-42)$ \\
\hline \multicolumn{2}{|c|}{$\begin{array}{l}\text { aOne patient had both BRCA1 and } 2 \text { mutation and is counted once in 'any BRCA mutation } \\
\text { cohort; one patient had a BRCAPro of } 68 \% \text { and is counted in 'any BRCA mutation' cohort } \\
\text { but not in either of 'BRCA1' or 'BRCA2' cohorts. } \\
\text { b Includes both carboplatin and cisplatin chemotherapy. One patient received } 3 \text { months o } \\
\text { oxaliplatin/gemcitabine/cyclophosphamide therapy before enrolment of our trial, bu } \\
\text { details were not available and excluded from the total platinum calculations. }\end{array}$} \\
\hline
\end{tabular}

study when the patients were given a specialised administration program.

Clinical parameters, signs, and symptoms, as well as clinical outcomes were evaluated for the 18 patients who developed HSR on trial. Those patients had a median of 2 cycles on study and 11.5 cumulative carboplatin cycles at the time of their initial on-trial HSR. The most common reaction symptoms were pruritus and rash $(89 \%$ and $78 \%$, respectively); other signs and symptoms are described in Table 2. A relatively similar proportion of patients experienced grades 1,2 , and 3 HSR on study (33\%, 39\%, and $28 \%$, respectively). Grade 3 reactions are defined as a prolonged reaction, recurrence of symptoms following initial improvement, and/or hospitalisation for clinical sequelae in the NCI CTCAE version 4.0. In addition to the common symptoms, many of these patients had one or more symptoms of chest tightness, hypotension, and dyspnea, and were managed by stopping carboplatin infusion and initiating intravenous fluids, ranitidine, diphenhydramine, and/or steroids with close monitoring in the ICU as deemed necessary.

In all, 17 of the 18 patients completed their infusion at the time of initial HSR on study after initiation of supportive management with intravenous diphenhydramine, ranitidine, and/or methylprednisolone as needed, followed by a slow-infusion protocol. Carboplatin was discontinued in one patient because of concurrent grade 2 elevated creatinine unresponsive to hydration. Seventeen patients with HSR on study were rechallenged on subsequent cycles, and four additional patients (total 5, 28\%) eventually had to discontinue carboplatin treatments before the eight cycle

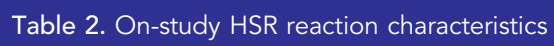

\begin{tabular}{|c|c|}
\hline & HSR on study \\
\hline & $\mathbf{N}=18(\% /$ range $)$ \\
\hline \multicolumn{2}{|l|}{ Grade } \\
\hline 1 & $6(33.3)$ \\
\hline 2 & $7(38.9)$ \\
\hline 3 & $5(27.8)$ \\
\hline \multicolumn{2}{|l|}{ Symptoms } \\
\hline Pruritus & $16(88.9)$ \\
\hline Rash & $14(77.8)$ \\
\hline Chest tightness & $7(38.9)$ \\
\hline Tachycardia & $6(33.3)$ \\
\hline Flushing & $5(27.8)$ \\
\hline Throat tightness & $5(27.8)$ \\
\hline Nausea/vomiting & $4(22.2)$ \\
\hline Hypotension & $4(22.2)$ \\
\hline Angioedema & $4(22.2)$ \\
\hline Dyspnea & $4(22.2)$ \\
\hline Delayed reaction & $1(5.6)$ \\
\hline Median carboplatin study cycle at time of initial HSR & $2(1-5)$ \\
\hline $\begin{array}{l}\text { Median cumulative carboplatin cycle at time of initial } \\
\text { HSR }\end{array}$ & $11.5(3-22)$ \\
\hline \multicolumn{2}{|l|}{ Outcome } \\
\hline Successfully continuing on study & $6(33.3)$ \\
\hline Off carboplatin because of HSR & $5(27.8)$ \\
\hline Off study for progression of disease & $5(27.8)$ \\
\hline Off study for other reasons: & $2(11.1)$ \\
\hline Elective withdrawal & $1(5.6)$ \\
\hline Intolerable adverse effects & $1(5.6)$ \\
\hline
\end{tabular}

maximum due to repeat grades 2-3 HSR despite premedications and slow infusion. Six (33\%) patients remain on trial and are continuing therapy per protocol following their HSR. Five (28\%) discontinued therapy because of progression of disease, one $(6 \%)$ patient for recurrent grade 3 neutropenia despite growth factor support, and one $(6 \%)$ voluntarily withdrew to participate in another clinical trial.

Risk factor comparison for carboplatin HSR. Previously reported risk factors for development of carboplatin HSR include age, history of allergy to medication and environmental factors, platinum-free interval, a single maximum dose of $>650 \mathrm{mg}$, and cumulative carboplatin exposure. We examined these risk factors as a function of HSR status (Table 3). The HSR group was further categorised into those who had HSR on trial and those with a previous history of HSR. The platinum-free interval and single maximum dose of $>650 \mathrm{mg}$ on the current trial were analysed only in the patients who developed HSR on trial in comparison with those with no history of HSR. No statistical difference was observed in the previously recognised potential risk factors when the groups with any history of HSR, HSR on trial, or HSR before trial, were compared with patients with no HSR. However, 93\% (27 out of 29) of patients with any history of HSR had a deleterious BRCA mutation, which was significantly higher than the 50\% (29 out of 58) mutation frequency seen in patients without a history of HSR $(P<0.0001)$. Statistical significance was retained in the HSR subgroups: $89 \%$ of patients with HSR on trial $(P=0.005)$ and $100 \%$ of patients with a prior history of HSR $(P=0.0002)$ had 


\begin{tabular}{|c|c|c|c|c|c|c|c|}
\hline & $\begin{array}{c}\text { No history of } \\
\text { any HSR }\end{array}$ & $\begin{array}{l}\text { Any history } \\
\text { of } \mathrm{HSR}^{\mathrm{a}}\end{array}$ & & HSR on study ${ }^{a}$ & & $\begin{array}{l}\text { HSR before } \\
\text { study }^{a}\end{array}$ & \\
\hline & $\mathbf{N}=\mathbf{5 8}(\% /$ range $)$ & $\mathbf{N}=\mathbf{2 9}(\% /$ range $)$ & $\boldsymbol{P}$-value ${ }^{\mathrm{b}}$ & $\mathbf{N}=18(\% /$ range $)$ & $\boldsymbol{P}$-value ${ }^{b}$ & $\mathbf{N}=15(\% /$ range $)$ & $P$-value ${ }^{b}$ \\
\hline Median age & $57(28-80)$ & $53(27-73)$ & 0.11 & $51(27-73)$ & 0.09 & $56(34-73)$ & 0.5 \\
\hline History of allergy & $26(44.8)$ & $18(62.1)$ & 0.17 & $9(50.0)$ & 0.79 & $10(66.7)$ & 0.16 \\
\hline \multirow{2}{*}{$\begin{array}{l}\text { Median platinum-free } \\
\text { interval in months }{ }^{c}\end{array}$} & 15.8 & NA & NA & 21.4 & 0.2 & NA & NA \\
\hline & $(5.7-82.4)$ & & & $(8.5-59.4)$ & & & \\
\hline $\begin{array}{l}\text { Single maximum dose } \\
>650 \mathrm{mg}^{c}\end{array}$ & $10(17.2)$ & NA & NA & $3(16.7)$ & 1 & NA & NA \\
\hline $\begin{array}{l}\text { Median number of prior } \\
\text { carboplatin cycles }\end{array}$ & $8(2-42)$ & $12(0-23)$ & 0.47 & $9(0-19)$ & 0.85 & $13(4-23)$ & 0.079 \\
\hline \multicolumn{8}{|l|}{ BRCA mutation status $^{d}$} \\
\hline $\begin{array}{l}\text { BRCA1 } \\
\text { BRCA2 } \\
\text { Any BRCA mutation }\end{array}$ & $\begin{aligned} 25 & (43.1) \\
5 & (8.6) \\
29 & (50.0)\end{aligned}$ & $\begin{array}{l}19(65.5) \\
7(24.1) \\
27(93.1)\end{array}$ & $\begin{array}{c}0.069 \\
0.095 \\
<0.0001\end{array}$ & $\begin{array}{l}11(61.1) \\
4(22.2) \\
16(88.9)\end{array}$ & $\begin{array}{c}0.28 \\
0.2 \\
0.005\end{array}$ & $\begin{array}{l}11(73.3) \\
4(26.7) \\
15(100)\end{array}$ & $\begin{array}{c}0.046 \\
0.079 \\
0.0002\end{array}$ \\
\hline \multicolumn{8}{|c|}{ 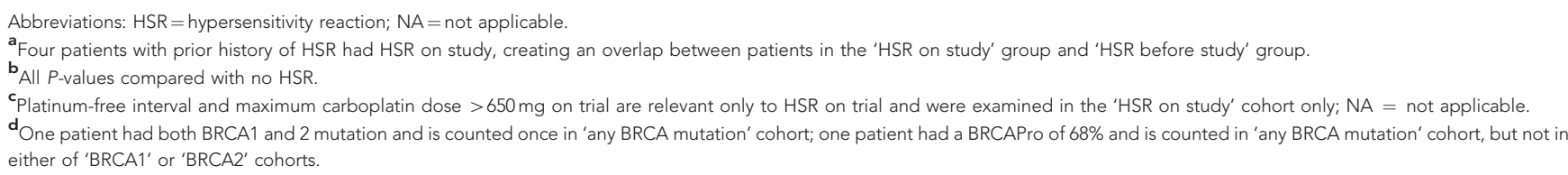 } \\
\hline
\end{tabular}

deleterious BRCA1/2 mutations. Patients with on-trial HSR were further subdivided based on the grade of their reaction, which still showed a statistically significantly higher proportion of patients with HSR of any grade having a BRCA mutation compared with those without a reaction $(P=0.027)$. When all patients were categorised based on BRCA mutation status, 48\% (27 out of 56) of patients with a BRCA mutation had a history HSR compared with 6\% (2 out of 31) in those without a BRCA mutation $(P<0.0001)$.

Adjustment for potential confounding variables. A multivariable analysis was conducted to examine the role of potentially confounding variables, as well as to measure the impact of carrying a deleterious BRCA1/2 ${ }^{\text {mut }}$ (Table 4). Age and number of prior carboplatin cycles (7-12 cycles) were not found to be independent risk factors. Nonsignificant risk was observed for previous history of allergy (odds ratio (OR) 2.3; 95\% confidence interval (CI): $0.82-6.4$ ) and prior carboplatin cycles greater than 12 (OR 2.2; 95\% CI: $0.65-7.6) . \mathrm{BRCA} / 2^{\text {mut }}$ status was identified as a strong and independent risk factor for HSR with an OR of 13.1 (95\% CI: 2.6-65.4; $P=0.0017$ ). The subset of patients who newly developed HSR on trial were analysed separately with all relevant risk factors including platinum-free interval and a single maximum carboplatin dose $>650 \mathrm{mg}$. Carrying a deleterious BRCA1/2 ${ }^{\mathrm{mut}}$ was an independent risk factor for HSR on study (OR 5.9; 95\% CI: 1.1-31.7; $P=0.039)$. These results identify deleterious germline mutation in BRCA1 or 2 as an unanticipated susceptibility factor for HSR to carboplatin.

Onset of carboplatin HSR and its impact on clinical outcome. We next hypothesised that carrying a deleterious BRCA1/2 ${ }^{\text {mut }}$ may not only increase susceptibility, but may also change the timing of the onset of HSR. The timing of the HSR could not be ascertained in four patients with a prior history of carboplatin HSR; those patients were excluded, leaving 52 BRCA1/2 ${ }^{\text {mut }}$ carriers and 31 control patients for analysis. The time of first documented platinum HSR was used for patients with more than one reaction, and patients without a HSR were censored at the cumulative carboplatin cycle number of their last infusion. Mutation carriers had a significantly earlier onset of HSR in response to carboplatin exposure (Figure $1, P=0.003$ ). Patients without a BRCA1/2 ${ }^{\mathrm{mut}}$ had a lower incidence of reaction after 20 cycles of carboplatin ( $8 \%$ vs $54 \%$ ). We then asked if developing a HSR any time during the patient's oncologic history affected clinical outcome on our study. No statistically significant difference in progression-free survival between patients with and without a history of carboplatin HSR was observed on the carboplatin-olaparib combination therapy (Figure 2A). In addition, no difference in progression-free survival was noted when BRCA $1 / 2^{\text {mut }}$ carriers were subgrouped based on HSR status (Figure 2B).

\section{DISCUSSION}

Carboplatin HSR is as an important adverse event in ovarian cancer patients who have repetitive exposure to platinum-based chemotherapy. Women with deleterious germline mutations in BRCA $1 / 2$ tend to retain platinum responsiveness and may live longer, such that they may have a greater lifetime platinum exposure compared with those without a mutation (Ben David et al, 2002; Chetrit et al, 2008; Alsop et al, 2012). We examined HSR risk in women enrolled on our carboplatin/olaparib studies, a cohort enriched for women with germline BRCA1/2 mutations. Our study demonstrates that a deleterious mutation or high BRCAPro score, a 'positive' BRCA1/2 ${ }^{\text {mut }}$ status, is an independent risk factor for development of carboplatin HSR. In addition to increased susceptibility, a deleterious BRCA1/2 ${ }^{\text {mut }}$ was associated with an earlier onset of HSR. The development of an allergic reaction to carboplatin, with its attendant potential for early carboplatin termination, did not adversely affect clinical benefit of the carboplatin/olaparib regimen in our 56 mutation carriers. This may have been influenced by the clinical activity of olaparib in mutation carriers and high-grade serous ovarian cancer patients (Fong et al, 2009; Gelmon et al, 2011). 
Table 4. Multivariable logistic regression analysis of HSR risk factors

\begin{tabular}{|c|c|c|c|c|c|c|}
\hline & \multicolumn{3}{|c|}{ Any history of HSR } & \multicolumn{3}{|c|}{ HSR on study } \\
\hline & Odds ratio & $95 \% \mathrm{Cl}$ & $\boldsymbol{P}$-value & Odds ratio & $95 \% \mathrm{Cl}$ & $P$-value \\
\hline \multicolumn{7}{|l|}{ Age } \\
\hline $\begin{array}{l}0-55 \\
55-70 \\
>70\end{array}$ & $\begin{array}{c}\text { Reference } \\
0.93 \\
1\end{array}$ & $\begin{array}{l}0.31-2.8 \\
0.13-8.2\end{array}$ & $\begin{array}{l}0.89 \\
0.98\end{array}$ & $\begin{array}{c}\text { Reference } \\
0.5 \\
0.89\end{array}$ & $\begin{array}{l}0.13-1.9 \\
0.10-7.7\end{array}$ & $\begin{array}{l}0.32 \\
0.92\end{array}$ \\
\hline \multicolumn{7}{|c|}{ History of allergy } \\
\hline $\begin{array}{l}\text { No } \\
\text { Yes }\end{array}$ & $\begin{array}{c}\text { Reference } \\
2.3\end{array}$ & $0.82-6.4$ & 0.12 & $\begin{array}{c}\text { Reference } \\
1.4\end{array}$ & $0.40-4.5$ & 0.64 \\
\hline \multicolumn{7}{|c|}{ Platinum-free interval ${ }^{a}$} \\
\hline $\begin{array}{l}\text { 0-12 Months } \\
\text { 12-24 Months } \\
>24 \text { Months }\end{array}$ & $\begin{array}{l}\text { NA } \\
\text { NA } \\
\text { NA }\end{array}$ & & & $\begin{array}{c}\text { Reference } \\
1.4 \\
2.6\end{array}$ & $\begin{array}{c}0.28-6.8 \\
0.49-14.1\end{array}$ & $\begin{array}{l}0.7 \\
0.26\end{array}$ \\
\hline \multicolumn{7}{|c|}{ Single maximum dose ${ }^{a}$} \\
\hline $\begin{array}{l}\leqslant 650 \mathrm{mg} \\
>650 \mathrm{mg}\end{array}$ & $\begin{array}{l}\text { NA } \\
\text { NA }\end{array}$ & & & $\begin{array}{c}\text { Reference } \\
1\end{array}$ & $0.19-5.6$ & 0.98 \\
\hline \multicolumn{7}{|c|}{ Prior carboplatin cycles } \\
\hline $\begin{array}{l}0-6 \\
7-12 \\
>12\end{array}$ & $\begin{array}{c}\text { Reference } \\
1.1 \\
2.2\end{array}$ & $\begin{array}{l}0.29-3.9 \\
0.65-7.6\end{array}$ & $\begin{array}{c}0.94 \\
0.2\end{array}$ & $\begin{array}{c}\text { Reference } \\
1.1 \\
1.9\end{array}$ & $\begin{array}{l}0.26-4.7 \\
0.41-8.5\end{array}$ & $\begin{array}{l}0.89 \\
0.42\end{array}$ \\
\hline \multicolumn{7}{|c|}{ BRCA mutation } \\
\hline $\begin{array}{l}\text { No } \\
\text { Yes }\end{array}$ & $\begin{array}{c}\text { Reference } \\
13.1\end{array}$ & $2.6-65.4$ & 0.0017 & $\begin{array}{c}\text { Reference } \\
5.9\end{array}$ & $1.1-31.7$ & 0.039 \\
\hline
\end{tabular}

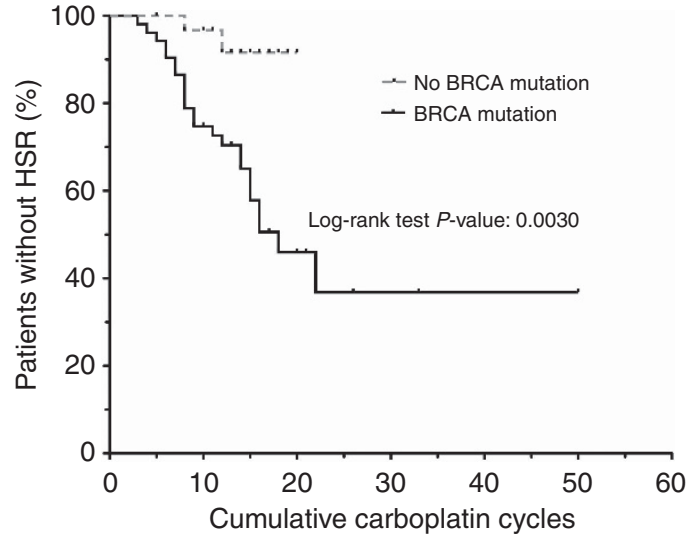

Figure 1. Kaplan-Meier analysis of the risk of development of HSR over cumulative cycles of platinum therapy. Patients are stratified by BRCA mutation status.

Cumulative exposure of carboplatin is the most wellcharacterised predictive factor for development of carboplatin hypersensitivity (Markman et al, 1999; Gadducci et al, 2008). HSR is rare in the first few exposures. Multiple studies have shown increasing incidence of HSR as cycle number increases, with a median of eight cycles at the time of first reaction (Markman et al, 1999; Rose et al, 2003). As first-line treatment is generally six cycles, HSR tends to occur early in the second carboplatin regimen in platinum-sensitive patients. We found that HSR actually occurred with fewer platinum cycles administered in BRCA mutation carriers. This was contrary to our original hypothesis that BRCA1/2 $2^{\text {mut }}$ carriers experienced more HSR because of greater overall platinum exposure. Other described risk factors include age $<70$ years (Joly et al, 2011), a history of allergy to environmental factors or medications (Markman et al, 2003; Gadducci et al, 2008), receiving single dose of carboplatin $>650 \mathrm{mg}$ (Sugimoto et al, 2011), and platinum-free interval $>12$ months (Schwartz et al, 2007), 13 months (Sugimoto et al, 2011), or 2 years (Gadducci et al, 2008). In our cohort, these risk factors were not predictive of HSR, although our findings may be somewhat limited by our study population size. Another potential contributor to carboplatin HSR is the use of combination therapy. Carboplatin administered with paclitaxel had a $33.1 \%$ frequency of HSR compared with carboplatin with pegylated liposomal doxorubicin $(15.5 \%, P<0.001)$ in the CALYPSO study (Joly et al, 2011). Our HSR incidence rate of $21 \%$ on trial is consistent with previously reported rates in the setting of recurrent ovarian cancer (Markman et al, 1999; Joly et al, 2011). We cannot dissect the role, if any, of olaparib on the frequency of HSR on trial since all patients received olaparib as part of the clinical trial regimen. There are no reports to date describing a possible increase in immunogenicity or hypersensitivity related to olaparib or other members of the PARP inhibitor class. Recent studies in murine asthma model, however, suggest that PARP1 inhibition may have anti-inflammatory effects by regulating IL-5 production (Datta 

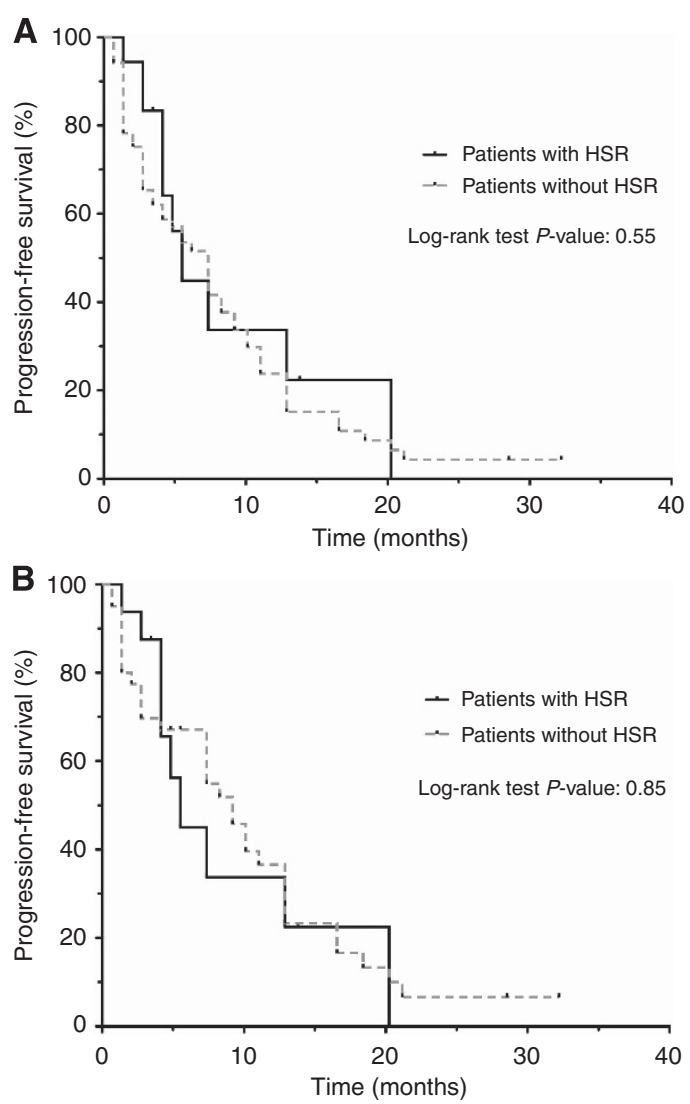

Figure 2. (A) Kaplan-Meier analysis of the progression-free survival from study entry for the 87 patients on study. (B) Kaplan-Meier analysis of the progression-free survival from study entry for the 56 patients with a positive BRCA mutation status.

et al, 2011). Thus, olaparib is unlikely to have contributed significantly to increase the HSR rates in our cohort of patients.

The clinical mechanism of hypersensitivity to platinum is unknown, but it is thought to be secondary to an IgE-mediated type I hypersensitivity or type IV T-cell-mediated hypersensitivity, particularly in delayed reactions (Makrilia et al, 2010). Carboplatin may act as a hapten that binds to serum proteins to elicit an immunologic reaction (Tonkin et al, 1993). Patients may be sensitised to the protein-platinum adduct beginning with the first-line treatment, with immunologic restimulation during subsequent regimens (Markman et al, 1999). Based on these findings, skin testing has been examined as a possible predictor of a reaction, but has not been shown to always reliably predict carboplatin HSR (Gomez et al, 2009). In addition to the platinum compounds bound to serum proteins, the DNA-platinum adduct itself may be immunogenic. Loss of normal BRCA function impairs homologous recombination required for error-free repair of DNA double-strand breaks that may occur secondary to torsion caused by or excision of interstrand platinum-DNA adducts (Farmer et al, 2005). It has been reported that dysfunctional BRCA1 also disrupts nucleotide excision repair, the mechanism through which platinum adducts are repaired (Husain et al, 1998). Thus, impaired DNA repair may contribute to increase in the DNA-platinum adducts, subsequently augmenting exposure and possible HSRs to carboplatin.

We observed no loss of clinical benefit from therapy in our BRCA1/2 ${ }^{\text {mut }}$ patients with a history carboplatin HSR before or on study. Frequently, patients who develop carboplatin HSR will have platinum therapy discontinued because of concerns of potential subsequent life-threatening anaphylaxis. Some patients may be challenged with a different platinum, such as cisplatin or oxaliplatin (Gutierrez et al, 2002; Jones et al, 2003). Approaches incorporating pre-treatment with histamine blockers and corticosteroids, with a progressive slow-infusion, can successfully manage reactions to administer additional carboplatin therapy (Rose et al, 2003; Confino-Cohen et al, 2005). Our study patients with a prior history of carboplatin HSR received methylprednisolone, ranitidine, and diphenhydramine, and followed a progressive slow-infusion protocol. With the application of this approach, 4 of the 15 patients who had a prior history of carboplatin HSR (27\%) developed HSR on trial, 3 of whom were successfully re-challenged with a modified slow-infusion protocol and received additional carboplatin doses. Only 5 of 18 patients (28\%) who reacted to carboplatin on trial discontinued carboplatin secondary to their HSR. This suggests that, with appropriate premedications and infusion strategies, most patients with a history of HSR can receive subsequent carboplatin infusions without further issues.

Potential weaknesses of our study include its single institution nature, study size, and patient recruitment to two studies with partial temporal overlap. Its retrospective nature prevented controlling for patient characteristics and possible confounders such as age, platinum-free interval, and number of prior carboplatin cycles. The treatment-defined dose of AUC 4 or 5 on trial meant that some patients, dosed for actual weight and creatinine $\left(\geqslant 0.6 \mathrm{mg} \mathrm{dl}^{-1}\right)$, may have received an absolute dose of carboplatin $>650 \mathrm{mg}$, one of the previously suggested risk factors. In all, 15 of the $87(17 \%)$ patients on study received at least one dose of carboplatin $>650 \mathrm{mg}$. However, there were no statistically significant differences in these parameters between those with or without carboplatin HSR in univariable and multivariable analysis.

Our study demonstrates that a deleterious BRCA1/2 mutation status is associated with a greater risk and earlier onset of carboplatin HSR, independent of other reported risk factors. Carboplatin re-treatment can be successful for ovarian cancer patients with prior history of HSR with appropriate premedications and a slow progressive infusion protocol. We suggest that careful observation for and management of HSR is necessary for BRCA1/2 mutation carriers because of this increased risk.

\section{ACKNOWLEDGEMENTS}

We thank Dr JoAnne Zujewski for her contributions in clinic and Dr Seth Steinberg for his inputs on the statistical analysis. We also thank I Prosak and $\mathrm{H}$ Minassie for their data management assistance. This work was supported by the Intramural Program of the Center for Cancer Research, National Cancer Institute, and olaparib was provided to the National Cancer Institute by AstraZeneca. DHM was funded through the NIH Medical Research Scholars Program, a public-private partnership supported jointly by the NIH and through the Foundation for the NIH (details on program and donors: http://www.fnih.org/ work/programs-development/medical-research-scholars-program).

\section{CONFLICT OF INTEREST}

The authors declare no conflict of interest.

\section{REFERENCES}

Alsop K, Fereday S, Meldrum C, deFazio A, Emmanuel C, George J, Dobrovic A, Birrer MJ, Webb PM, Stewart C, Friedlander M, Fox S, Bowtell D, Mitchell G (2012) BRCA mutation frequency and patterns of treatment response in BRCA mutation-positive women with ovarian cancer: a report from the Australian Ovarian Cancer Study Group. J Clin Oncol 30: 2654-2663. 
Ben David Y, Chetrit A, Hirsh-Yechezkel G, Friedman E, Beck BD, Beller U, Ben-Baruch G, Fishman A, Levavi H, Lubin F, Menczer J, Piura B, Struewing JP, Modan B (2002) Effect of BRCA mutations on the length of survival in epithelial ovarian tumors. J Clin Oncol 20: 463-466.

Burger RA, Brady MF, Bookman MA, Fleming GF, Monk BJ, Huang H, Mannel RS, Homesley HD, Fowler J, Greer BE, Boente M, Birrer MJ, Liang SX (2011) Incorporation of bevacizumab in the primary treatment of ovarian cancer. $N$ Engl J Med 365: 2473-2483.

Chetrit A, Hirsh-Yechezkel G, Ben-David Y, Lubin F, Friedman E, Sadetzki S (2008) Effect of BRCA1/2 mutations on long-term survival of patients with invasive ovarian cancer: the national Israeli study of ovarian cancer. J Clin Oncol 26: 20-25.

Confino-Cohen R, Fishman A, Altaras M, Goldberg A (2005) Successful carboplatin desensitization in patients with proven carboplatin allergy. Cancer 104: 640-643.

Datta R, Naura AS, Zerfaoui M, Errami Y, Oumouna M, Kim H, Ju J, Ronchi VP, Haas AL, Boulares AH (2011) PARP-1 deficiency blocks IL-5 expression through calpain-dependent degradation of STAT- 6 in a murine asthma model. Allergy 66: 853-861.

Farmer H, McCabe N, Lord CJ, Tutt AN, Johnson DA, Richardson TB, Santarosa M, Dillon KJ, Hickson I, Knights C, Martin NM, Jackson SP, Smith GC, Ashworth A (2005) Targeting the DNA repair defect in BRCA mutant cells as a therapeutic strategy. Nature 434: 917-921.

Fong PC, Boss DS, Yap TA, Tutt A, Wu P, Mergui-Roelvink M, Mortimer P, Swaisland H, Lau A, O'Connor MJ, Ashworth A, Carmichael J, Kaye SB, Schellens JH, de Bono JS (2009) Inhibition of poly(ADP-ribose) polymerase in tumors from BRCA mutation carriers. N Engl J Med 361: 123-134.

Gadducci A, Tana R, Teti G, Zanca G, Fanucchi A, Genazzani AR (2008) Analysis of the pattern of hypersensitivity reactions in patients receiving carboplatin retreatment for recurrent ovarian cancer. Int J Gynecol Cancer 18: 615-620.

Gelmon KA, Tischkowitz M, Mackay H, Swenerton K, Robidoux A, Tonkin K, Hirte H, Huntsman D, Clemons M, Gilks B, Yerushalmi R, Macpherson E Carmichael J, Oza A (2011) Olaparib in patients with recurrent high-grade serous or poorly differentiated ovarian carcinoma or triple-negative breast cancer: a phase 2, multicentre, open-label, non-randomised study. Lancet Oncol 12: 852-861.

Gomez R, Harter P, Luck HJ, Traut A, Kommoss S, Kandel M, du Bois A (2009) Carboplatin hypersensitivity: does introduction of skin test and desensitization reliably predict and avoid the problem? A prospective single-center study. Int J Gynecol Cancer 19: 1284-1287.

Gutierrez M, Pautier P, Lhomme C (2002) Replacement of carboplatin by oxaliplatin may be one solution for patients treated for ovarian carcinoma who are hypersensitive to carboplatin. J Clin Oncol 20: 353.

Husain A, He G, Venkatraman ES, Spriggs DR (1998) BRCA1 up-regulation is associated with repair-mediated resistance to cis-diamminedichloroplatinum(II). Cancer Res 58: 1120-1123.

Joly F, Ray-Coquard I, Fabbro M, Donoghoe M, Boman K, Sugimoto A, Vaughan M, Reinthaller A, Vergote I, Ferrandina G, Dell'Anna T, Huober J, Pujade-Lauraine E (2011) Decreased hypersensitivity reactions with carboplatin-pegylated liposomal doxorubicin compared to carboplatin-paclitaxel combination: analysis from the GCIG CALYPSO relapsing ovarian cancer trial. Gynecol Oncol 122: 226-232.

Jones R, Ryan M, Friedlander M (2003) Carboplatin hypersensitivity reactions: re-treatment with cisplatin desensitisation. Gynecol Oncol 89: 112-115.

Koshiba H, Hosokawa K, Kubo A, Miyagi Y, Oda T, Watanabe A, Honjo H (2009) Incidence of Carboplatin-related hypersensitivity reactions in
Japanese patients with gynecologic malignancies. Int J Gynecol Cancer 19: 460-465.

Lee CW, Matulonis UA, Castells MC (2004) Carboplatin hypersensitivity: a 6-h 12-step protocol effective in 35 desensitizations in patients with gynecological malignancies and mast cell/IgE-mediated reactions. Gynecol Oncol 95: 370-376.

Makrilia N, Syrigou E, Kaklamanos I, Manolopoulos L, Saif MW (2010) Hypersensitivity reactions associated with platinum antineoplastic agents: a systematic review. Met Based Drugs 2010: 1-10.

Markman M, Kennedy A, Webster K, Elson P, Peterson G, Kulp B, Belinson J (1999) Clinical features of hypersensitivity reactions to carboplatin. J Clin Oncol 17: 1141.

Markman M, Zanotti K, Kulp B, Peterson G (2003) Relationship between a history of systemic allergic reactions and risk of subsequent carboplatin hypersensitivity. Gynecol Oncol 89: 514-516.

Morgan JS, Adams M, Mason MD (1994) Hypersensitivity reactions to carboplatin given to patients with relapsed ovarian carcinoma. Eur J Cancer 30A: 1205-1206.

Ozols RF, Bundy BN, Greer BE, Fowler JM, Clarke-Pearson D, Burger RA, Mannel RS, DeGeest K, Hartenbach EM, Baergen R (2003) Phase III trial of carboplatin and paclitaxel compared with cisplatin and paclitaxel in patients with optimally resected stage III ovarian cancer: a Gynecologic Oncology Group study. J Clin Oncol 21: 3194-3200.

Polyzos A, Tsavaris N, Kosmas C, Arnaouti T, Kalahanis N, Tsigris C, Giannopoulos A, Karatzas G, Giannikos L, Sfikakis PP (2001) Hypersensitivity reactions to carboplatin administration are common but not always severe: a 10-year experience. Oncology 61: 129-133.

Rose PG, Fusco N, Smrekar M, Mossbruger K, Rodriguez M (2003) Successful administration of carboplatin in patients with clinically documented carboplatin hypersensitivity. Gynecol Oncol 89: 429-433.

Schwartz JR, Bandera C, Bradley A, Brard L, Legare R, Granai CO, Dizon DS (2007) Does the platinum-free interval predict the incidence or severity of hypersensitivity reactions to carboplatin? The experience from Women and Infants' Hospital. Gynecol Oncol 105: 81-83.

Sugimoto H, Iwamoto T, Murashima Y, Tabata T, Sagawa N, Okuda M (2011) Risk factors contributing to the development of carboplatin-related delayed hypersensitivity reactions in Japanese patients with gynecologic cancers. Cancer Chemother Pharmacol 67: 415-419.

TCGARNetwork (2011) Integrated genomic analyses of ovarian carcinoma. Nature 474: 609-615.

Tonkin KS, Rubin P, Levin L (1993) Carboplatin hypersensitivity: case reports and review of the literature. Eur J Cancer 29A: 1356-1357.

USPSTF (2005) Genetic risk assessment and BRCA mutation testing for breast and ovarian cancer susceptibility: recommendation statement. Ann Intern Med 143: 355-361.

Vergote I, Trope CG, Amant F, Kristensen GB, Ehlen T, Johnson N, Verheijen RH, van der Burg ME, Lacave AJ, Panici PB, Kenter GG, Casado A, Mendiola C, Coens C, Verleye L, Stuart GC, Pecorelli S, Reed NS (2010) Neoadjuvant chemotherapy or primary surgery in stage IIIC or IV ovarian cancer. N Engl J Med 363: 943-953.

This work is published under the standard license to publish agreement. After 12 months the work will become freely available and the license terms will switch to a Creative Commons AttributionNonCommercial-Share Alike 3.0 Unported License. 